\title{
Entfernung eines eingekapselten Cysticercus aus dem Auge.
}

\author{
Von \\ Dr. Stolting, Augenarzt in Hannover. \\ Mit einem Holzschnitt.
}

Am 17. August dieses Jahres wurde mir anf Veranlassung des Herrn Stabsarztes Dr. Benzler hier ein zwanzigjähriger Soldat zugeführt, welcher über Abnahme seines Sehvermögens auf dem rechten Auge klagte. Schmerzen oder Reizzustände waren nie vorhanden gewesen; genauere Angaben über partielle Verdunkelungen des Gesichtsfeldes wurden keine gemacht. Die Diagnose, dass Patient wahrscheinlich an einem subretinal sitzenden Cysticercus leide, war von dem genannten Herrn bereits gemacht.

Die Prüfung des V. ergab 1. E. $\mathbf{S}=1$, r. Fingerzählen bei leicht excentrischer Fixation auf $1,5 \mathrm{~m}$.

Bei der hochgradigen Herabsetzung der Sehschärfe war ich erstaunt, völlig durchsichtige Medien vor mir zu haben. Ausser einigen wenigen Glaskörperopacitäten bestand die einzige Veränderung in der Anwesenheit einer Cyste nahe dem hintern Pol des Auges mit daneben liegender Chorioidealnarbe. Diese Cyste mit ibrem grössten Durchmesser meridional gestellt, hatte eine Länge von $7-8 \mathrm{~mm}$, eine Breite von $4-5 \mathrm{~mm}$; ihr hinterer am meisten dem Opticus genäherter $\mathrm{P}_{0} \mathrm{l}$ war von demselben noch $6 \mathrm{~mm}$ entfernt. Von der Papille aus lag die Neubildung nach aussen oben, so zwar, dass der Augenhintergrund als Kreis aufgefasst, eine das äussere obere Segment desselben halbirende 
Linie die untere Längsseite derselben berülrt haben würde. An der Oberfläche dieses in den Glaskörper vorspringenden blasigen Gebildes konnte man einen leichten Einkniff constatiren, etwa yon der Mitte der oberen Längsseite ausgehend, welcher die Cyste in zwei fast gleich grosse Theile sonderte, ohne übrigens irgendwie tiefer einzudringen. Nicht abhüngig von dieser Schnürfurche (sit venia verbo) war die Farbe des Gebildes, nämlich gelblich weiss in der ganzen Ausdehnung der Oterfläche, mit Ausnahme einer einzigen peripher nach ausson oben gelegenen Parthie, welche blendend weiss und selhnig glänzend das licht reflectirte. Hier auch fehlten völlig dio sonst auf der Oberfläche ziemlich reichlich vorhandenen Gefässe. Letztere als im Zusammenhang mit dem System der Netzhautgefässe zu crkennen gelang nicht, was deshalb nicht Wunder nebmen darf, als die überhängenden Ränder der Blase die Umschlagstelle verdeckten.

Undulirende Bewegungen an irgend einom Theil aufzufinden, war mir nicht möglich, doch will ich nicht verschweigen, dass es dem anfänglich behandelnden Dr. Benzler gelungen war, solche Vibrationen zu sehen. Einige wenige Glaskörperfäden hingen der Oberfläche an und flottirten bei Bewegungen des Auges. Amotio retinae nicht sichtbar.

Nebcn dieser Cyste weiter nach aussen oben, etwa $2 \mathrm{~mm}$ davon entfernt, befand sich in der Chorioidea eine Narbe von dreieckiger Form und fast der Grösse der Blase selbst. Innerhalb derselben fehlte das Pigment und erschienen zahlreiche neugebildete Gefässe. Narbe und Cyste waren durch einen Strich atrophischer Chorioidea von $0,5 \mathrm{~mm}$ Breite verbunden.

Den Befund in anderer Weise zu deuten, als bereits gesehehen, nämlich dass man es mit der bindegewebigen Kapsel eines Entozoon zu thun habe, welche zum grössten Theil von Netzhaut überzogen sei, erschien auch mir nicht zulässig. Namentlich unterstützte die Auffassung, dass es sich um ein Entozoon handeln müsse, die Chorioidealnarbe und der von derselben auslanfende, unter der Cyste endende Gang. Auffallend blieb die Starrbeit der Cystenwand, sowie die absolute Undurchsichtigkeit auch an der Stelle nach oben, wo, wie man aus dem Bilde schliessen musste, die Umbüllung unter der Netzhant zu Tage trat.

Nach Verlauf weniger Wochen hatte sich das Bild nur insoweit geändert, als jetzt auch an der untern Längsseite bei 
einer mässigen allgemeinen Vergrösserung der Blase eine solche sehnige gefässlose Partie sichtbar wurde, was mich zu der Annabme verleitete, dass die Blase locker unter der Netzhaut liege und vielleicht in Kürze ganz frei in den Glaskörper hineinragen resp. sich lösen werde.

Der Eingriff ersehien bei der Lage der Dinge nnaufschieblich. Ich empfahl dem Patienten, sich bis zur Operation möglichst ruhig zu verhalten und schritt am 28. August unter Assistenz der Herren Dr. Benzler und Dr. Bruns zur Entfernung.

Es bedarf heute, nach den zahlreichen Veröffentlichungen Graefe's und Leber's wohl kaum noch der Erorterung, in welcher Weise die Operation vorgenommen wurde, nur soviel sei erwähnt, dass der Rect. ext. in der von Graefe angegebenen Nanier tenotomirt wurde und dass die Sehne des frei zu präparirenden Scleralbogens, wie sich durch Rechnung und Construction ergab, $19 \mathrm{~mm}$ betrug.

Nach Anlegung des Schnittes und Erweiterung desselben durch einen Scheerenschlag hoffte ich spontan die Blase sich entbinden zu sehen. Diese Hoffnung wurde jedoch getäuscht, auch das Eingelen mit einem scharfen Häckchen war nicht von besserem Erfolg begleitet and ich entschloss mich, ehe noch mehr Glaskörper verloren ginge, die Wunde wieder zu schliessen. Zu diesem Vorgehen bewog mich hauptsächlich die vorgefasste Meinung, dass die Cyste sich sehr leicht entbinden werde, als aber dieselbe mit dem ziemlich reichlich ausfliessenden Glaskörper nicht entwickelt wurde, als anch das vorsichtig eingeführte Häckchen ans der Wunde nichts hervorbrachte, musste ich auf den Gedanken kommen, mit dem Schnitte weit gefehlt zu haben. Mein Erstaunen war daher nicht gering, als ich am achten Tage, zum ersten Male ophthalmoscopirend, sah, wie der Schnitt unmittelbar (vielleicht $1 \mathrm{~mm}$ ) neben der Cyste nach oben lag. Einige Blntflocken von geringem Umfang lagen zugleich auf der neuen Narbe und der Cyste. Diese letztere hatte genau das gleiche Aussehen wie früher.

Selbstverständlich wurde die Wiederholnng der Operation in Aussicht genommen, um so mehr, als der Eingriff auf das Sehvermögen kaum einen Einfluss gehabt hatte. Es konnten noch Finger in $1 \mathrm{~m}$ bei excentrischer Fixation gezählt werden.

Die Heilung ging ungemein schnell von statten. Beim ersten Verbandwechsel nach drei Tagen schon hatte die Pupille 
auf einen Tropfen Atropin reagirt, wach 14 Tagen wurde Patient vorläufig entlassen, nach weiterem Verlaufe von zwei Wochen zum zweiten Male operirt. Bei dieser zweiten Operation kam mir ungemein zu statten, dass ein Zweifel über die Wahl der Einsehnittstelle nicht bestehen konnte und so gelang es denn auch schnell, ebenfalls nach Verlängerung des Schnittes mit der Scheere die Cyste zwischen die Branchen einer Irispincette zu fassen. Auch jetzt ging die Entbindung nicht so ohne Weiteres, sondern es bedurfte eines sehr energischen Zuges und der Hinzunahme einer zweiten stärkeren Pincette, um die Cyste heraus zu befördern. Im letzten Moment des Anziehens platzte dabei die Wand und in dieser Rupturstelle wurde ein zweites diaphanes Bläschen sichtbar, welches, bei weiterem Anziehen ebenfalls zerspringend, als feines Häutchen hängen blieb. Letzteres Häutchen erwies sich als die Cysticercusblase; durch leichten Zug an demselben entwickelte sich nämlich der Kopf des Wurmes $\mathrm{S}$ förmig gekrümmt und in sein Receptaculum eingestiulpt.

Die Maasse der Cysticercusblase selbst anzugeben ist leider nicht möglich, da dieselbe geplatzt war, dagegen beträgt die Laange vom Kopf und Halstheil zusammen, obne die Sförmige Krümmung zo reehnen, $2 \mathrm{~mm}$. Saugnäpfe und Hakenkranz sind sichtbar.

Die in Wasser gelegte bindegewebige Umhüllung nahm sofort wieder ihre bei der Compression mit der Pincette verloren gegangene kugelige Gestalt an nnd ich erkannte, dass ihre Basis flach und dunkel pigmentirt war. Es hatte sich also die Chorioidea von der Sclera gelöst und war dem Zuge gefolgt, dabei war jedoch nicht mehr von dieser Membran verloren gegangen, als eben die Basis der Blase deckte. Die so entfernte halbkugelige Cyste wurde nun in Serienschnitte zerlegt und für die mikroskopisehe Untersuchung vorbereitet, deren Resultat hier folgen möge, nur sei einer Besonderheit, welche beim Zerlegen des Präparates sich zeigte, auch hier gleich Er. wähnung gethan. Es erwies sich nämlich die Umbüllung als zur Hälfte zweikammerig, so nämlich, dass entsprechend dem oben erwähnten, an der obern Längsseite verlaufenden Finkniff eine Leiste in das Innere der Cyste vorsprang, welche ungefär bis in die Mitte ihres Lumens reichte.

Die Wand der kugeligen Kapsel bestand nun nicht, wie wir angenommen hatten - das Mikroskop liess auf den ersten 
Blick den Irrthum erkennen - aus neugebildetem Bindegewebe chorioidealen Ursprungs, sondern war einfach degenerirte Netzhaut mit den zum Theil noch dentlich erhaltenen Elementen derselben; namentlich konnte man die beiden Körnersehichten und die Fasersehicht erkennen, ebenso die Stäbehen- und Zapfenschicht, welch' letztere, als aus nervösen Elementen zusammengesetzt, zwar nicht mehr angesprochen, wohl aber noch dureh radiäre Zeichnung in ihrer Bedeutung verstanden werden konnte. Die Gefässe, welche alle in der äussern Hälfte der Cystenwand verliefen, waren zum Theil erhalten und mit Blutkörperehen gefült, zum Theil dureh Kernwucherungen obliterirt. Die Dicke der Cystenwand betrug im Mittel 0,48 mm, sie verdickte sich bis auf $0,7 \mathrm{~mm}$ dort, wo ein grosses Gefäss verlief, und verdünnte sich an der der Basis nahe liegenden Rupturstelle bis auf $0,1 \mathrm{~mm}$. Als Boden der Cyste, wo dieselbe von freier Chorioidea begrenzt wurde, erwies sich nur eine $2 \mathrm{~mm}$ im Durchmesser betragende Stelle, für die Grösse der Cyste gewiss

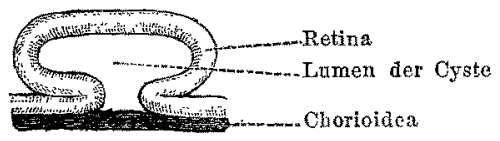

eine schmale Basis. Dieselbe wurde jedoch verbreitert durch die umgeschlagene und wiederum verwachsene Retina (s. Abb.). Die Dícke der Chorioidea an dieser Stelle mass $0,5 \mathrm{~mm}$. Hier sowohl als unter der verwachsenen Netzhaut war dieselbe von zahlreichen Rundzellen durchsetzt.

Die Heilung verlief auch nach dieser zweiten, weit eingreifenderen Operation ungemein günstig. Der erste Verbandwechsel, am 6. Tage vorgenommen, zeigte ein reizfreies Auge. Ein Tropfen Atropin genügte, die Pupille mittelweit zu machen. Leider war trotzdem der Rest des vorbandenen Sehvermögens erloschen, eine totale Ablösung der Netzhant war die Folge des Eingriffes gewesen. Sehr dentlich sah man die Stelle, wo die Cyste gesessen hatte, als breiten weissen glänzenden Placque. Bei einer späteren, am 24. October stattfindenden Vorstellung im ärztlichen Verein zeigte es sich dann, dass in der Zwischenzoit eine mässige Glaskörperblutung stattgefunden hatte, welche jedoch nicht verhinderte, wenn auch muihsam, jetzt noch die Narbe der Chorioidea zu erkennen. Dio Tension war normal, das Auge völlig reizfrei, leicht divergent. 
In zweierlei Beziehung scheint mir die soeben mitgetheilte Krankengeschichte von besonderem Interesse zu sein, nämlich durch die eigenthümlich feste Einkapselung des Entozoon, welche als durchaus ungewöhnlich bezeichnet werden muss und durch die Schwierigkeit, welche daraus fur die Operation erwuchs. Hätte ich mich nicht auf eine verbältnissmässig leichte Entwickelung gefasst gemacht, so wäre gewiss bei der günstigen Lage des Schnittes schon der erste Eingriff von Erfolg gewesen. $O b$ damit allerdings eine Spur von Sehvermögen, um mehr konnte es sich bei dem damaligen Zustande und bei der Entfernung eines grossen Stücks fast centraler Netzhaut nicht handeln, erhalten geblieben wäre, erscheint wohl mit Recht sehr zweifelhaft.

Nachtrag. Bei einer Vorstellung am 20. November ist der Glaskorper wieder soweit anfgehellt, dass die Narbe der Chorioidea leicht gesehen werden kann; Auge völlig reizfrei, nicht divergent. T. normal. 